\title{
Study on circular loop source TEM with different solutions
}

\author{
Laifu Wen, JiulongCheng, Jizhe Jia,Jin Zhou \\ College of Geoscience and Surveying Engineering \\ China University of Mining \&Technology, Beijing \\ Beijing, China \\ Wenlaifu0@163.com
}

\begin{abstract}
The circular loop source is calculated by the method ofinscribed polygon inner the circle or numerical calculation. The former uses decomposed polygon in each side with electric dipole, then stack all the electric dipole response; the latter adopts the method of digital filter to calculate the integral expression directly. There is some error between above methods and exact solutions in transient electromagnetic response values.This paper compares the two kinds of method. First, the equivalence of inscribed polygon is analyzedand the number of edges of the polygon, the proportioncoefficient of transceiver distance and the length of the dipole are debated. Second, the contrast research in homogeneous half space with the two methodsis carried on. Finally, layered medium is inversed by Artificial Bee Colony (ABC) algorithm and studying the fitting error. The results show that if inscribed polygonhas few numbers of edges, the response error and fitting error calculated by the method ofinscribed polygon inner the circle are higher than numerical calculation method in any conditions. But when the edge of inscribed polygon reach a certain number, the calculation accuracy is better than the numerical calculation methodunder a suitable parameter combination.
\end{abstract}

Keywords-circular loopsource; inscribed polygon inner the circle; parameter combination; numerical calculation; inversion of layered medium

\section{INTRODUCTION}

Transient electromagnetic method (TEM)is widely used in time domain electromagneticdetection problems; the shape of transmitter loop is mainly about rectangularloop ${ }^{[1]-[9]}$ and circular loop ${ }^{[10][11]}$. For the circular loop, there are two computing methods. Based on the above work, further study is carriedon inscribed polygon inner the circley,regard the proportioncoefficient of transceiver distance and the length of the dipole as a function of the number of polygon edges and the concept of the smallest proportion coefficient with different number of edges is put forward. Under this premise, optimal parameter combination is selected to calculateelectromagnetic response.Using the two methods respectively tocalculateelectromagnetic response in the homogeneous half space and using the two methods respectively to solve the inverse problem with Artificial Bee Colony algorithm in layered model. Comprehensive all the results, advantages and disadvantages of different approaches are acquired.

Natural Science Foundation of China (Grand No. 51574250) ; Specialized Research Fund for the Doctoral Program of Higher Education (No. 20120023110014)

\section{IITHE FUNDAMENTAL THEORY}

\section{A. The Minimum Proportion Coefficient}

The basic idea of inscribed polygon inner the circle as follows: according to cyclotomy in math, when the number of edges increases, the circumference of polygon is close to the perimeter of the circle. Therefore, inscribed polygon inner the circle can be used for a circular loop. Inside the loop, the electromagnetic field can be regarded as the combination of electromagnetic field from each side of the loop. When each side of the loop can be decomposed into multiple horizontal electric dipoles, the electromagnetic field at any point inside the loopcan be regarded as superposition of many horizontal electric dipoles.

In homogeneous half space, the vertical magnetic field of polygon can be obtained by horizontal electric dipole:

$$
\begin{gathered}
h_{z m}=\sum_{i=1}^{m} \sum_{j=1}^{n} h_{z j} \\
=\sum_{i=1}^{m} \sum_{j=1}^{n} \frac{I d s y}{4 \pi r^{3}} \times\left[\left(1-\frac{3}{2 \theta^{2} r^{2}}\right) \operatorname{erf}(\theta r)+\frac{3}{\theta r \sqrt{\pi}} e^{-\theta^{2} r^{2}}\right]
\end{gathered}
$$

Assuming that $R$ is the radius, $m$ is the number of edge, $L$ is the length of side and $d$ is the distance between loop center and each edge. So, there are the follow relations:

$$
\begin{aligned}
& L=2 R \sin (\pi / m) \\
& d=R \cos (\pi / m)
\end{aligned}
$$

Set each edge can be decomposed into $n$ electric dipoles.

$$
n=L / d s \geq 1
$$

Set

$$
A_{\min }=r_{\min } / d s=d / d s
$$

So

$$
A_{\min } \geq \cot (\pi / m) / 2
$$

Select $m=4,8,16,32,64,128,256,512$, we can obtain the curve of $A_{\min }=\cot (\pi / m) / 2$ (Fig. 1) and different $A_{\min }$ corresponding with $m\left(0\right.$, integrate $\left.A_{\min }\right)$. 
are significant differences in results obtained by different

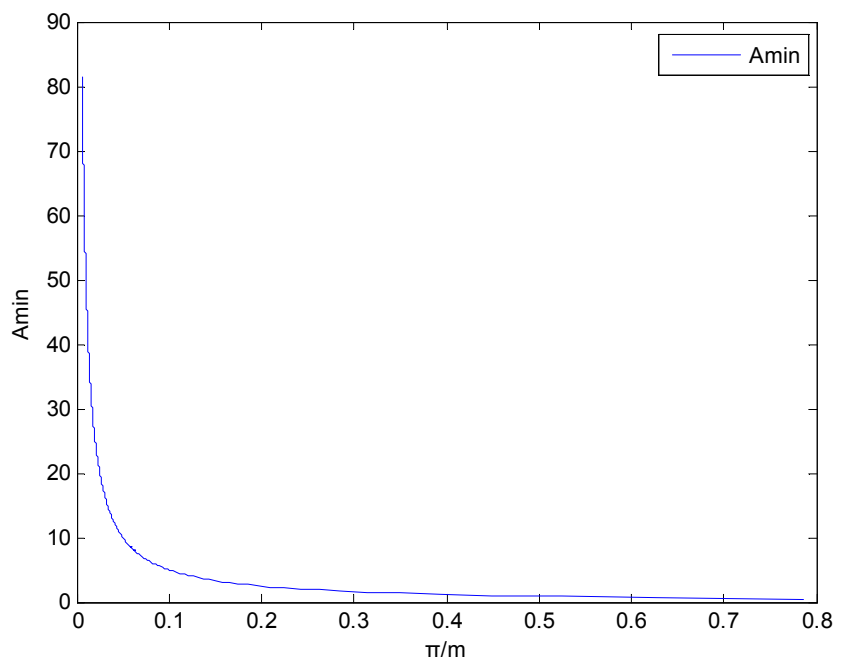

Fig. 1. The curve of $A_{\min }=\cot (\pi / m) / 2$

TABLE I. THE MinimumProportion COEFFICIENT

\begin{tabular}{|l|l|l|l|l|l|l|l|l|}
\hline$m$ & 4 & 8 & 16 & 32 & 64 & 128 & 256 & 512 \\
\hline$A_{\min }$ & 1 & 2 & 3 & 6 & 11 & 21 & 41 & 82 \\
\hline
\end{tabular}

TABLE II. TheOptimal Combination Between $m$ And $A$

\begin{tabular}{|c|c|c|c|c|c|c|c|c|}
\hline$m$ & 4 & 8 & 16 & 32 & 64 & 128 & 256 & 512 \\
\hline$A$ & 100 & 50 & 5 & 10 & 20 & 30 & 50 & 100 \\
\hline
\end{tabular}

B. The Optimal Combination

The analytical expressions of magnetic field deduced the circular loop in homogenous half space can be written as ${ }^{[7]}$ :

$$
h_{z}=\frac{I}{2 R}\left[\frac{3}{\sqrt{\pi} \theta R} e^{-\theta^{2} R^{2}}+\left(1-\frac{3}{2 \theta^{2} R^{2}}\right) \operatorname{erf}(\theta R)\right]
$$

As a general rule, the bigger $A$ is, the better the results is under the premise of 0 . But compared with analytic solution, there is an optimal $A$ corresponding to $m$ (TABLE II. ).

\section{The Method of Numerical Calculation}

The vertical magnetic field intensity in the center of receiver can be written as ${ }^{[11]}$ :

$$
H_{z}=I a \int_{0}^{\infty} \frac{\lambda^{2}}{\lambda+u_{1}} J_{1}(\lambda a) d \lambda
$$

Uses of Fast Hankel transform ${ }^{[13]}$ to calculate the Bessel function in (8) and Gaver-Stehfest inverse Laplace transform ${ }^{[14]}$ to realize the Frequency-Time Transformation.

\section{COMPARISION IN HOMOGENOUS HALF SPACE}

Set $\rho=100 \Omega \bullet m, r=50 m, I=1 A$, and the following results are calculated according to TABLE II. .

Fig. 2 shows in the observation period, the attenuation curves from different methods have the same trend. And there

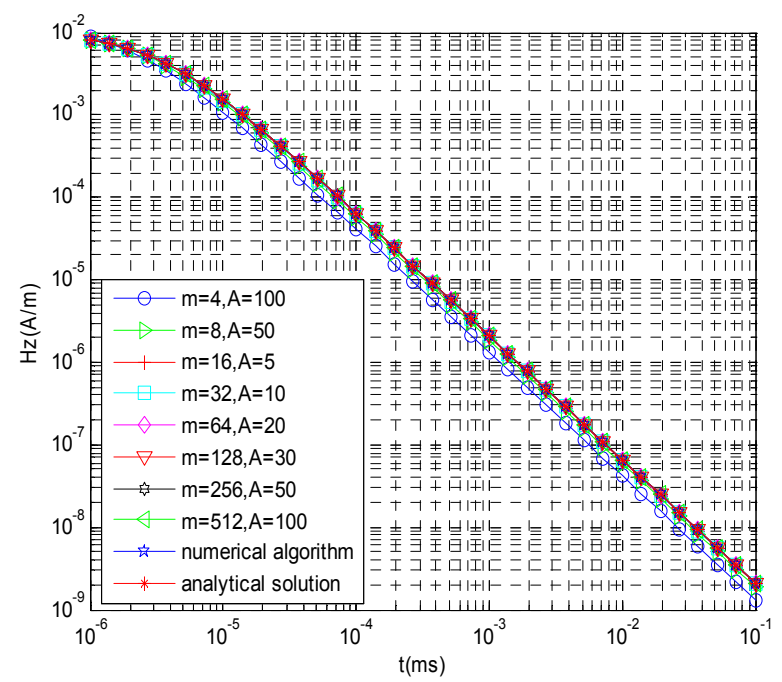

Fig. 2. The vertical magnetic fieldintensity

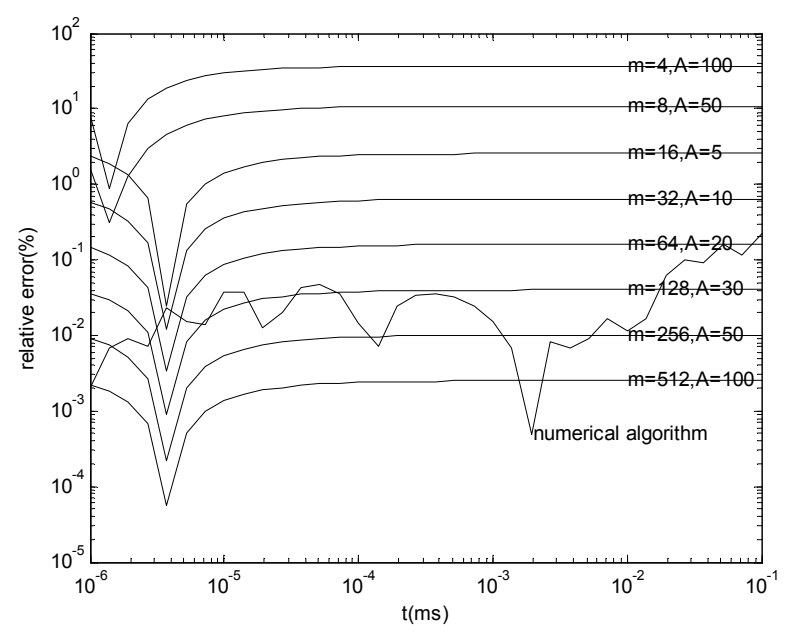

Fig. 3. The relative error of vertical magnetic fieldintensity

combination.Fig. 3 shows the vertical magnetic field intensity obtained by the method of numerical calculation maintains high accuracy in the observation time and the range of relative error is $3 \times 10^{-4} \% \sim 2 \times 10^{-1} \%$. For the method of inscribed polygon inner the circle, the precision enhances with the increase of the number of the polygon. Compared with the method of numerical calculation, if the number of edges of the polygon is less than a certain number (128), the calculated precision obtained was inferior to the numerical method. But if it reaches a certain number (256), the calculated precision obtained was superior to the numerical method as long as choose an appropriatecombination of parameters. 
Setsthe $\mathrm{H}$ type geoelectric model for example (0) and layered medium is inversed by Artificial Bee Colony algorithm (ABC). In order to make the results more representative, $m=128, A=30$ and $m=256, A=50$ are selected to calculate the results of the method of inscribed polygon inner the circle. The inversion results and curves of relative error as followed.

Oshows that the relative error of each parameter with numerical algorithm is $0.135 \%, 1.2 \%, 0.103 \%, 0.54 \%, 1.26 \%$ and the method of inscribed polygon inner the circle is $0.242 \%$, $8.71 \%, 0.258 \%, 1.88 \%, 9.43 \%$, while $m=128, A=30$ and $0.014 \%, \quad 0.99 \%, \quad 0.597 \%, \quad 0.064 \%, \quad 1.48 \%$, while $m=256, A=50$. Combined with the relative error of inversion result in Fig. 4, we can found that the numerical calculation method has a higher fitting precision before $10^{-2} \mathrm{~ms}$ than the method of inscribed polygon inner the circle when $m \leq 128$ and almost the same level in late time period, but the latter is lower in fitting precision in general within the calculate time. While $m \geq 256$, the latter has a higher fitting precision in general and this is same as the conclusion obtained from homogenous half space.

TABLE III. THE INVERSION RESULTS OF H TYPE GEOELECTRIC MODEL

\begin{tabular}{|l|l|l|l|l|l|}
\hline Parameters & $\rho_{1}$ & $\rho_{2}$ & $\rho_{3}$ & $h_{1}$ & $h_{2}$ \\
\hline $\begin{array}{l}\text { Theoretical } \\
\text { value }\end{array}$ & 100 & 10 & 300 & 150 & 50 \\
\hline Hunting zone & $50-150$ & $5-15$ & $\begin{array}{l}200- \\
400\end{array}$ & $\begin{array}{l}100- \\
200\end{array}$ & $10-100$ \\
\hline $\begin{array}{l}\text { Inversion of } \\
\text { numerical } \\
\text { algorithm }\end{array}$ & 100.135 & 10.120 & $\begin{array}{l}299.6 \\
91\end{array}$ & $\begin{array}{l}149.18 \\
5\end{array}$ & 50.629 \\
\hline $\begin{array}{l}\text { Inversion of } \\
A=128 \\
A=30\end{array}$ & 100.242 & 10.871 & $\begin{array}{l}299.2 \\
27\end{array}$ & $\begin{array}{l}147.17 \\
6\end{array}$ & 54.716 \\
\hline $\begin{array}{l}\text { Inversion of } \\
m=256 \\
A=50\end{array}$ & 99.986 & 10.099 & $\begin{array}{l}301.7 \\
91\end{array}$ & $\begin{array}{l}149.90 \\
3\end{array}$ & 50.739 \\
\hline
\end{tabular}

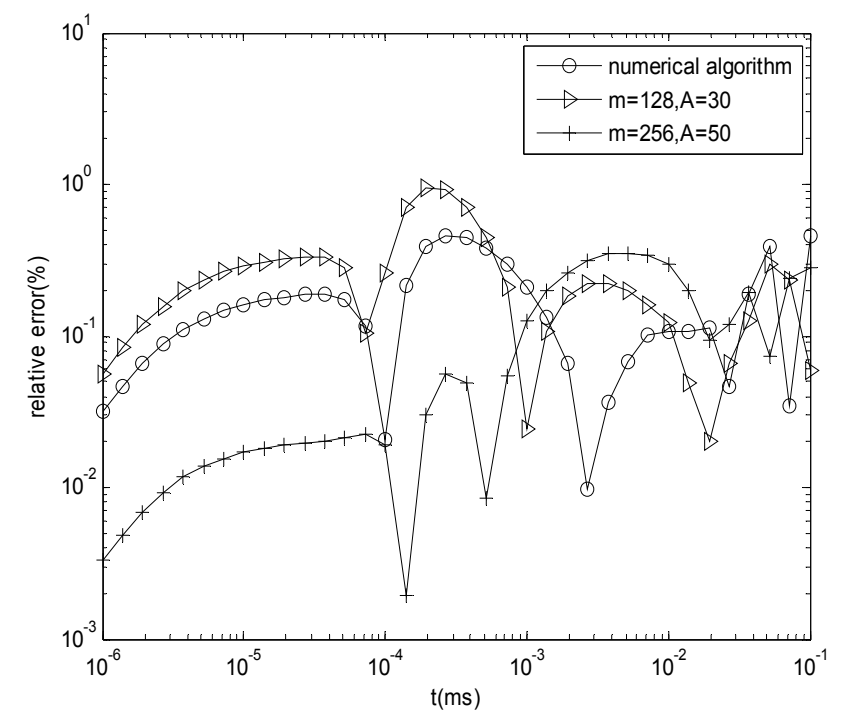

Fig. 4. The relative error of inversion result for magnetic field intensity

\section{CONCLUSIONS}

For the method ofinscribed polygon inner the circle, $m$ and Amust be combined. There is the smallest proportion coefficient $A$ and the optimal combination between $m$ and $A$.

For the method ofinscribed polygon inner the circle, the more number of polygon edges, the better accuracy of the results.

Under the condition of $m \leq 128$, thefitting error calculated by the method ofinscribed polygon inner the circle are higher than numerical calculation method; while $m \geq 256$, the former's calculation accuracy is better.

\section{ACKNOWLEDGMENT}

The research presented in this paper is supported byNatural ScienceFoundation of China (Grand No.51574250) and Specialized Research Fund for the Doctoral Program ofHigher Education (No.20120023110014).

\section{REFERENCES}

[1] PoddarM., Arectangular loop source of current on a multilayered earth: Geophysics, 1983,vol. 48, pp.107-109.

[2] Goldman M. M., Fitterman D. V.,Direct time-domain calculation of thetransientresponse for a rectangular loopover a two-layer medium: Geophysics, 1987,vol.52, pp.997-1006.

[3] Li J. P., Li T. L., Zhao X. F. and Liang T. M., Study on the TEM alltime apparent resistivity of arbitrary shape loop source over the layered medium: Progress in Geophysics (in Chinese), 2007, vol.22, pp.17771780 .

[4] Li J.P., Li T.L., Zhang Y.D., Tem forward and inversion of arbitrary shape loop source in layered media:Geophysical \& Geochemical exploration, 2012, vol.2, pp.256-259.

[5] Shi X. X., Yan S., Fu J. M., and Chen M. S., Improvement forinterpretation of central loop transient electromagnetic method: Chinese J. Geophys.(in Chinese), 2009,vol.52,pp.1931-1936.

[6] Li J. H., Liu S. C., Zhu Z. Q., et al., Relationship between electromagnetic field and magnetic field's symmetric excited by rectangular loop: Journal of Central South University (Science and Technology) (in Chinese), 2010,vol.41,pp.638-642.

[7] Zhou N. N., Xue G. Q., Li M. F., and Yan S., Response of large-loop TEM based on approximation of electric dipole: Coal geology\&exploration,2011, vol.4, pp.49-54.

[8] Xue G. Q., Zhou N. N., Yan S., Theoretical study on the errors caused by dipole hypothesis of large-loop TEM response: Chinese J .Geophys.(in Chinese), 2011, vol. 54,pp.2389-2396.

[9] XinH. C., Tang J. T., Xu Z. M., Finite-difference modeling of 2.5-D Transient Electromagnetic: Progress in Geophysics (in Chinese), 2014,vol. 29, pp.2278-2286.

[10] SINGH N P, MOGI T. Electromagnetic response of a large circular loop source on a layered earth: A new computation method[J]. Pure and Applied Geophysics, 2005, vol.162, pp.181-200.

[11] Xue G. Q., Li X., Guo W. B.,and Di Q. Y., Characters of response of large-loop transient electromagnetic field: OGP, 2007,vol.42,pp.586590.

[12] Nabighian M N, ed. Electromagnetic Methods in Applied Geophysics, Vol.1, Theory Society of Exploration Geophysicists (in Chinese). Beijing; Geological Publishing House, 1992.

[13] D.Guptasarma, B.Singh, New digital linear filters for Hankel J0 and J1 transforms: Geophysical Prospecting, 1997,vol.45, pp.745-762.

[14] Piao H. R., Yin C. C., Calculations of transient E.M. sounding using the GAYER-STEHFEST inverse Laplace transform method. Computing Techniques For Geophysical And Geochemical Exploration, 1987, vol.9,pp.296-302. 